\title{
Voltage control of magnetism in ferromagnetic structures
}

\author{
L. Herrera Diez ${ }^{a}$, W. Lin ${ }^{c}$, A. Bernand-Mantel ${ }^{a}$, L Ranno $^{a}$, D. Givord ${ }^{a}$, L. Vila ${ }^{b}$, Patrick

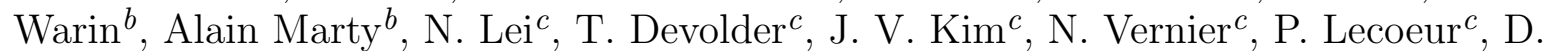 \\ Ravelosona ${ }^{c}$ \\ ${ }^{a}$ Institut Néel - CNRS-UJF, 25 avenue des Martyrs, F-38042 Grenoble Cedex 9, France \\ ${ }^{b}$ INAC/CEA Grenoble, 17 avenue des Martyrs, 38054 Grenoble Cedex 9, France \\ ${ }^{c}$ Institut d'Electronique Fondamentale, CNRS, UMR 8622, 91405 Orsay, France
}

\begin{abstract}
Until now, spintronics devices have relied on polarized currents, which still generate relatively high dissipation, particularly for nanodevices based on DW motion. A novel solution to further reduce power consumption is emerging, based on electric field (E) gating to control the magnetic state. Here, we will describe the state of the art and our recent experiments on voltage induced changes in the magnetic properties of ferromagnetic metals. A thorough description of the advances in terms of control of intrinsic properties such as magnetic anisotropy and ferromagnetic transition temperature as well as in intrinsic properties like coercive field and domain wall motion will be presented. Additionally, a section will be dedicated to the summary of the key aspects concerning the fabrication and performance of magneto-electric field-effect devices.
\end{abstract}

Keywords: Magnetic anisotropy, electric-field effect, magnetic domain wall, magneto-electric devices

\section{INTRODUCTION}

Spin-electronics is a prolific field of research that has many times transcended the limits of the basic science laboratories into widely spread technological applications. In particular, the ever-growing need for more efficient data storage and retrieval has fostered a constantly evolving activity in the area of non-volatile memories based on spintronics spin-valve structures. ${ }^{1,2}$ Great scientific efforts made in order to reduce the power consumption in MRAMs (magnetic random access memories) have led to the development of prototypes for spin-transfer-torque random access memories (STT-RAMs) where the soft layers of the spin-valve are manipulated by the use of spin-polarized currents. ${ }^{3,4}$ However, the spin-polarized current densities needed so far to operate STT-MRAM prototypes are still too high to be of commercial interest. Consequently, finding new paths for switching magnetic units and reducing the critical switching currents in magnetic tunnel junctions (MTJs) are nowadays a very active field of research. Voltage induced changes in the magnetic properties of technologically relevant ferromagnetic systems is an emerging and a seemingly promising route to magnetic-field free magnetization reorientation and the reduction of critical currents in spin-torque based devices. In the following, the most relevant achievements in the field of voltage control of magnetism will be introduced.

\subsection{Voltage control of magnetism}

Pioneering work on the realization of devices with voltage controlled magnetic properties can be dated more than one decade back. The observation of carrier-mediated ferromagnetism in diluted magnetic semiconductor (DMS) systems, namely, the ferromagnetic coupling between localized magnetic moments via an antiferromagnetic coupling with the hole charge carriers, presented a promising scenario for the creation of magnetic field-effect structures. Supported by this principle a spin field-effect transistor based on (Ir,Mn)As was realized and demonstrated a gate voltage induced transition temperature modulation. ${ }^{5}$ However, further development in DMS based devices could not avoid reaching a plateau since the transition temperatures in these materials have remained stubbornly low for practical applications. ${ }^{6}$

A step forward was made by the experimental demonstration of electric-field induced changes in coercive field of

E-mail: liza.herreradiez@grenoble.cnrs.fr, dafine.ravelosona@u-psud.fr

Spintronics V, edited by Henri-Jean Drouhin, Jean-Eric Wegrowe, Manijeh Razeghi,

Proc. of SPIE Vol. 8461, 84610Y - @ 2012 SPIE · CCC code: 0277-786/12/\$18 · doi: 10.1117/12.982039

Proc. of SPIE Vol. $846184610 Y-1$ 
over $4 \%$ in ultra-thin films of FePt and FePt, two metallic and technologically relevant ferromagnetic materials. ${ }^{7}$ In this case the success of the experiment was given by the strong electric field created by a polymer electrolyte gate, a significant surface magnetic anisotropy contribution with respect to the bulk anisotropy and a large surface to volume ratio of the magnetic films. At the heart of this promising effect is the fact that electric fields can induce a modulation of the electronic density close to the Fermi level which in itinerant ferromagnets corresponds to the density of $d$-electrons intimately linked to the magnetic properties. The surface of the magnets is the most practically accessible region to produce these changes in the electronic density, therefore, it is crucial that surface effects translate into the properties of the entire film and not become outshined by the bulk properties. This requirement can be fulfilled in materials where the surface anisotropy is large with respect to the bulk anisotropy and where the dimensions of the magnet assure a large surface to volume ratio.

The successful experiments in FePt and FePd have led to research activities involving other types of magnetic materials and device geometries. Ultra-thin iron films incorporated into solid-state metal/oxide/conducting electrode field-effect devices have shown to be susceptible to magnetic anisotropy changes as a function of the gate voltage. $^{8}$ Similarly, in $\mathrm{CoFeB}$ films the magnetic anisotropy and the coercive field can be manipulated with electric fields. ${ }^{9}$ Thin films of cobalt not only show important voltage-induced changes in the coercive field but also a significant modulation of the ferromagnetic transition temperature which is proposed as the basis of a potential 'field-effect magnet' that could be electrically driven into and out of the ferromagnetic state. ${ }^{10,11}$ Regarding the potential uses of the voltage control of magnetism in magnetic memories the electric field assisted switching from the low to high resistance state in $\mathrm{CoFeB} / \mathrm{MgO} / \mathrm{CoFeB}$ magnetic tunnel junctions stands out as a significant step forward. Aided by the presence of a constant bias magnetic field below coercivity the $\mathrm{CoFeB}$ layers can be selectively and reversibly switched by a voltage pulse since the values of the coercive field of the two ferromagnetic layers shift in opposite directions for a given sign of the gate voltage. The current densities involved in these electric-field assisted switchings are several orders of magnitude smaller than those typically employed in spin transfer torque switching. ${ }^{12}$

An equally interesting aspect of the voltage assisted magnetization reversal can be studied at the level of domain wall dynamics, where the electric field control of magnetic domain wall propagation and nucleation may bring new perspectives for applications in domain wall logic devices. ${ }^{13}$ Experiments involving magnetic imaging have shown that the charge accumulation at the surface of the ferromagnet can modify the energy barriers involved in domain wall propagation and consequently increase or decrease the domain wall velocity in the creep regime. ${ }^{14-16}$ The modulation of the domain wall velocity has been shown to depend exponentially on the gate voltage applied. The question of the electric-field effect on domain nucleation still remains open and is an important aspect in view of the potential development of voltage-driven switching units and the design of single domain devices. Kerr microscopy studies in ultra-thin films of CoPt show that the electric-field assisted modulation of the magnetic anisotropy has a large impact not only on the domain wall propagation but also on the number of domains involved in the magnetization reversal. ${ }^{17}$

Voltage control of magnetism can not only occur via a direct carrier-density modulation mechanism but also via the susceptibility of the magnetic properties to mechanical stress. This was demonstrated in devices consisting of hybrid ferromagnetic/piezoelectric structures. ${ }^{18,19}$ In this systems the voltage induced strain served as a means to achieve easy axis reorientation and control of domain wall propagation in $(\mathrm{Ga}, \mathrm{Mn}) \mathrm{An}$ and $\mathrm{CoFeB}$ devices, respectively.

\section{MAGNETIC FIELD-EFFECT DEVICE FABRICATION AND OPERATION}

In order to produce an electric field and to also measure simultaneously a signal that can account for the influence of this electric field on magnetism Hall bar structures with integrated top gate electrodes were fabricated. A schematic cross section of the device along the source-drain axis is shown in Fig. 1. The continuous ferromagnetic film is first patterned into Hall bar structures by photolithography and ion beam etching which is followed by the deposition of $\mathrm{Au}$ contacts. In a second step a dielectric layer is added to the entire device by atomic layer deposition (ALD) followed by the deposition of the top gate electrode which finalizes the fabrication process. From a technical point of view the success of the voltage control of magnetic properties relies on creating the largest possible charge accumulation per unit area at the surface of the ferromagnet. This is undoubtedly related to the limits in the voltage that can be applied to the top gate electrode without damaging the device 


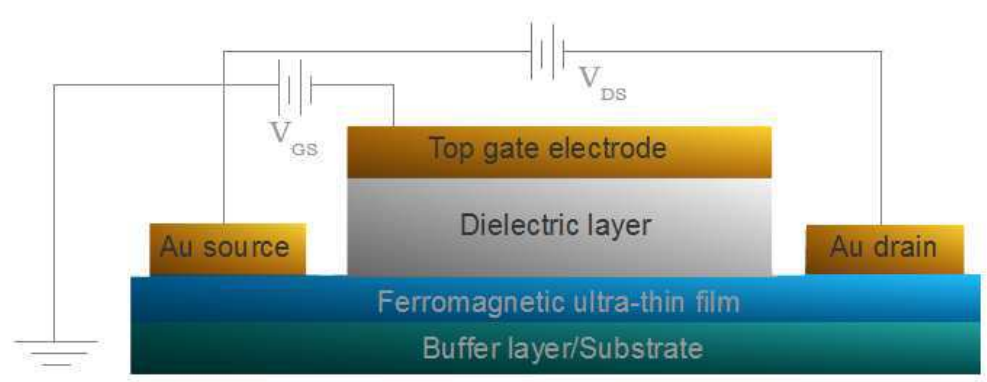

Figure 1. Magnetic field-effect device geometry. The ferromagnetic film is patterned into Hall bar structures where the main channel between source and drain can be biased with a top gate voltage applied across a dielectric layer.

Table 1. Dielectric constants $\left(\epsilon_{r}\right)$ of materials commonly used in the fabrication of field-effect structures.

\begin{tabular}{|l|l|}
\hline \multicolumn{1}{|c|}{ Material } & \multicolumn{1}{|c|}{$\epsilon_{r}$} \\
\hline $\mathrm{SiO}_{2}$ & 3.9 \\
\hline $\mathrm{Al}_{2} \mathrm{O}_{3}$ & 9 \\
\hline $\mathrm{MgO}$ & 9.9 \\
\hline $\mathrm{HfO}_{2}$ & 25 \\
\hline $\mathrm{ZrO}_{2}$ & 25 \\
\hline
\end{tabular}

but also to the polarization efficiency of the dielectric layer separating the gate and ferromagnetic electrodes. This is better visualized in the following expression for the amount of charges accumulated $Q$ per unit surface $S: Q / S=\epsilon_{r} \epsilon_{0} E=\epsilon_{r} \epsilon_{0} V / d$ where $\epsilon_{r}$ and $\epsilon_{0}$ are the dielectric constants of the dielectric layer and that of vacuum, respectively, $\mathrm{E}$ is the electric field, $\mathrm{V}$ is the applied gate voltage and $\mathrm{d}$ is the separation between the top gate and ferromagnetic electrodes (namely, the thickness of the dielectric layer). For a given gate voltage the electric-field effect will scale with the value of $\epsilon_{r}$ which accounts for the efficiency of the charge polarization inside the dielectric. In table 1 the values of the dielectric constants of different materials used in field effect structures are listed. ${ }^{20}$

Applying a given gate voltage to the magneto-electric device will have a much larger impact on the magnetic properties if, for example, $\mathrm{SiO}_{2}$ can be replaced by $\mathrm{HfO}_{2}$ as the dielectric material. It is worth mentioning that the values of the dielectric constants are highly susceptible to growth conditions and to the final thickness of the grown layer. ${ }^{20,21}$ Additionally, materials with high dielectric constant like $\mathrm{HfO}_{2}$ may show a resistance switching behavior that could affect its performance in a field effect device. ${ }^{22}$ For this reason it is of great importance to thoroughly characterize the properties of the dielectric barrier before incorporating it into the magnetic devices.

The interaction of the dielectric layer with the surface of the magnetic material also needs to be analyzed in detail in zero gate voltage conditions. As mentioned, the magnetic anisotropy of the ferromagnetic material is highly susceptible to surface modifications and therefore the nature of the ferromagnet/dielectric interface can have a large impact on the magnetic properties of the film even in the absence of an applied gate voltage. Fig. 2 shows the effect of the deposition of an $\mathrm{ALD} \mathrm{HfO}_{2}$ layer on the surface of a $\mathrm{Pt}(3.5 \mathrm{~nm}) / \mathrm{Co}(0.5 \mathrm{~nm}) / \mathrm{Pt}(1$ $\mathrm{nm})$ film. The critical reduction in the coercive field as well as in the remanence value of the Kerr rotation loops upon deposition of the $\mathrm{HfO}_{2}$ layer indicate a large modification of the magnetic anisotropy of Co.

In epitaxial FePt films where the surface anisotropy is less prominent than in $\mathrm{Co} / \mathrm{Pt}$ layers the addition of $\mathrm{HfO}_{2}$ or $\mathrm{ZrO}_{2}$ does not modify significantly the magnetic anisotropy at zero gate voltage, an increase (decrease) in anisotropy can only be achieved by the action of a negative (positive) gate voltage. ${ }^{24}$ However, as seen in Fig. 3, the electric-field effect in coercivity that can be observed using $\mathrm{HfO}_{2}$ (a) and $\mathrm{ZrO}_{2} / \mathrm{HfO}_{2}$ (b) dielectric layers is completely suppressed when using $\mathrm{Al}_{2} \mathrm{O}_{3}$. This effects holds for both ALD (Fig. 3 (c)) and in-situ (not shown) grown $\mathrm{Al}_{2} \mathrm{O}_{3}$. It is worth mentioning that the poor performance of the devices containing $\mathrm{Al}_{2} \mathrm{O}_{3}$ layers is not due to the difference in dielectric constant with respect to $\mathrm{HfO}_{2}$ and $\mathrm{ZrO}_{2}$. The electric fields in 


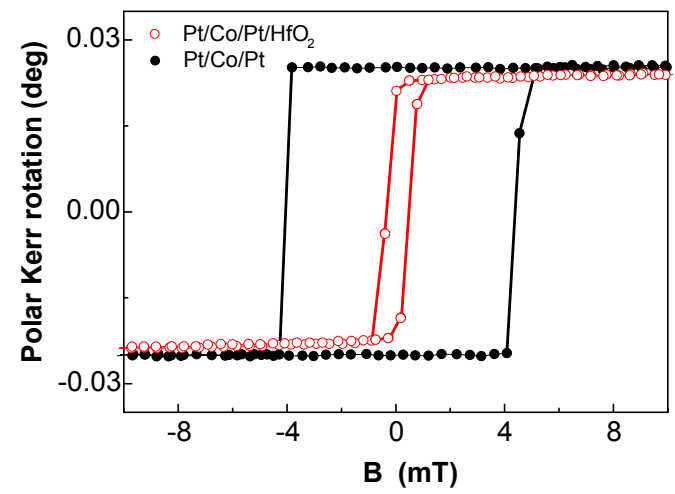

Figure 2. Kerr rotation as a function of the magnetic field for $\mathrm{Pt} / \mathrm{Co} / \mathrm{Pt}$ films before and after deposition of an $\mathrm{ALD}_{\mathrm{HfO}}$ dielectric layer. The changes at the surface of the ferromagnetic material due to the incorporation of $\mathrm{HfO}_{2}$ have a large impact on the value of the coercive field and remanence.
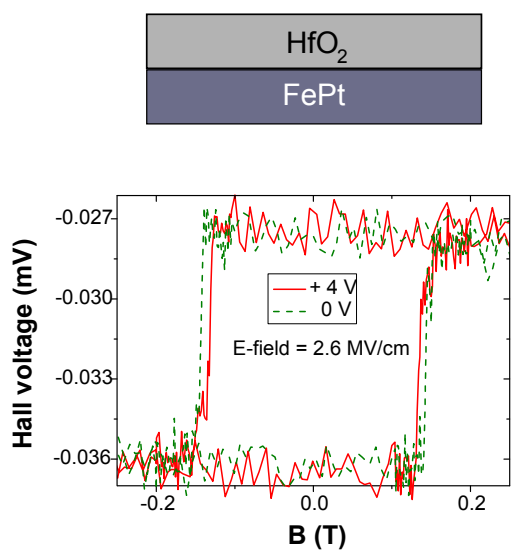
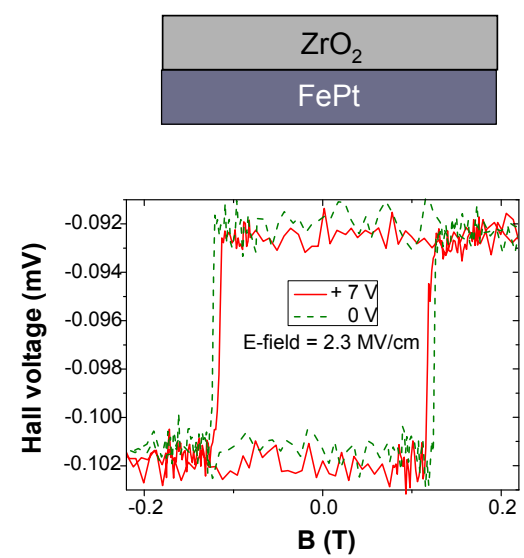
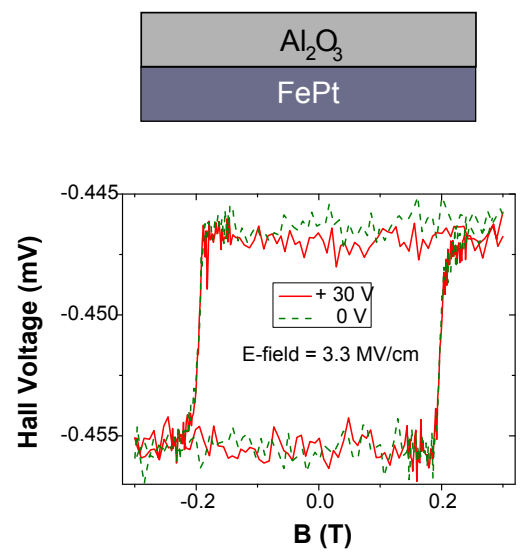

Figure 3. Hall voltage vs. magnetic field plots under gate voltages of $+4 \mathrm{~V}$ (a), $+7 \mathrm{~V}$ (b), and $+30 \mathrm{~V}$ (c) in FePt devices where the ferromagnet was in direct contact with dielectric layers of $\mathrm{HfO}_{2}, \mathrm{ZrO}_{2}$ and $\mathrm{Al}_{2} \mathrm{O}_{3}$, respectively. The electricfield effect in coercivity that can be achieved with $\mathrm{FePt} / \mathrm{HfO}_{2}$ and $\mathrm{FePt} / \mathrm{ZrO}_{2}$ interfaces is suppressed in $\mathrm{FePt} / \mathrm{Al} \mathrm{O}_{3}$ interfaces. 

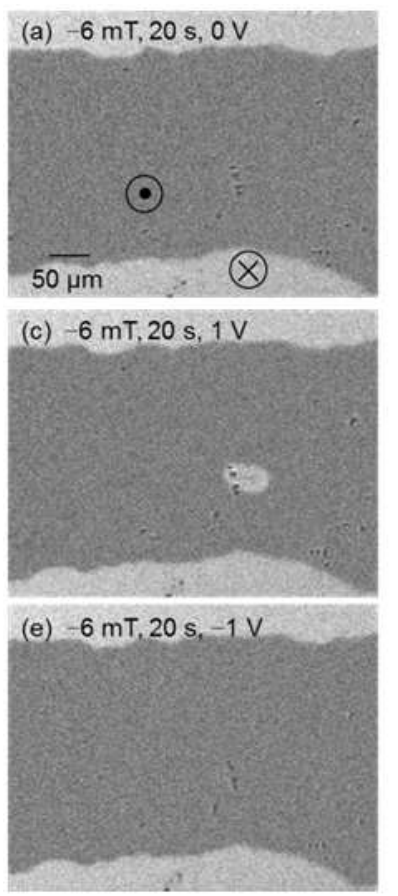
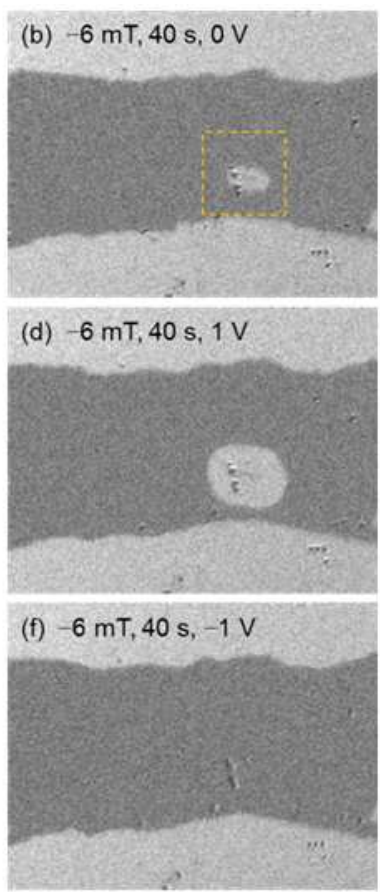

Figure 4. Polar Kerr microscopy images of magnetic domains in a $\mathrm{Pt} / \mathrm{Co}(0.5) / \mathrm{Pt}(0.5) / \mathrm{Al} 2 \mathrm{O} 3(50) / \mathrm{ITO}$ structure under gate voltages of $0 \mathrm{~V}, 1 \mathrm{~V}$ and $-1 \mathrm{~V}$, respectively. All images were taken after saturating in a positive magnetic field and applying a negative $6 \mathrm{mT}$ magnetic field pulse. The dot and cross labels indicate the positive magnetization pointing out of the film surface and the negative one pointing into the film, respectively. The dash square indicates the magnetic domain influenced strongly by the gate voltage.

the $\mathrm{HfO}_{2}$ (a) and $\mathrm{ZrO}_{2} / \mathrm{HfO}_{2}$ devices that produced the results in Fig. 3 (a) and (b) were $2.6 \mathrm{MV} / \mathrm{cm}$ and $2.3 \mathrm{MV} / \mathrm{cm}$, respectively, and the electric field at which the first clear evidence of a coercivity change is seen is close to $1.3 \mathrm{MV} / \mathrm{cm}\left(2 \mathrm{~V}\right.$ gate voltage for the $\mathrm{HfO}_{2}$ device). In the $\mathrm{Al}_{2} \mathrm{O}_{3}$ device the hysteresis loop under gate voltage was recorded with an electric field of $3.3 \mathrm{MV} / \mathrm{cm}$ which compensates the difference in the dielectric constant. A similar effect has been observed by other authors in sputtered FePt films where a visible effect of gate voltage on coercivity can be seen with a $\mathrm{MgO} / \mathrm{Al}_{2} \mathrm{O}_{3}$ layer while it completely disappears when using only a pure $\mathrm{Al}_{2} \mathrm{O}_{3}$ layer. ${ }^{23}$ These findings exemplify that in the selection of the dielectric layer both the perspectives of maximizing the charge accumulation at a given gate voltage and also the preservation of the intrinsic properties of the magnetic material must be taken into account.

\section{ELECTRIC FIELD-EFFECT IN DOMAIN WALL DYNAMICS}

The voltage induced changes in coercive field discussed earlier are expected to have an impact on the magnetic field driven magnetization reversal also at the level of domain nucleation and domain wall motion. Fig. 4 shows polar Kerr microscopy images of the expansion of a magnetic domain in a $\mathrm{Pt}(5 \mathrm{~nm}) / \mathrm{Co}(0.5 \mathrm{~nm}) / \mathrm{Pt}(0.5$ $\mathrm{nm}) / \mathrm{Al}_{2} \mathrm{O}_{3}(50 \mathrm{~nm})$ structure terminated by a transparent ITO top gate electrode. These measurements were carried out after saturating in a positive magnetic field and applying a negative $6 \mathrm{mT}$ magnetic field pulse. The time delay of the selected frames with respect to the moment of the application of the field pulse is $20 \mathrm{~s}$ and $40 \mathrm{~s}$ for the images in the left and right column, respectively. The device was set to operate at gate voltages of $0 \mathrm{~V}$ (top), $+1 \mathrm{~V}$ (middle) and $-1 \mathrm{~V}$ (bottom).

The expansion of the magnetic domain that is highlighted by a dotted square presents a marked dependence on the gate voltage applied. For a gate voltage of $+1 \mathrm{~V}$ the domain wall propagation is favoured as shown by the increased size of the magnetic domain at both 20 s and 40 s with respect to the measurements done at $0 \mathrm{~V}$. Following 
the same trend, the measurements done under a gate voltage of $-1 \mathrm{~V}$ show the opposite effect, the expansion of the magnetic domain is critically hindered and provokes the suppression of the action of the nucleation center since no switching activity is observed neither at 20s nor at 40s. These results illustrate how the expected changes in the magnetic anisotropy translate into changes not only in coercivity but also in domain wall dynamics. The observed trend of the electric-field effect suggests that positive charges accumulating at the Pt/Al2O3 interface enhance magnetic domain wall propagation in the underlaying Co layer, while negative accumulated charges hinder domain wall nucleation and/or propagation. This in turn can be correlated to a weakening (strengthening) of the magnetic anisotropy for positive (negative) applied gate voltages, in agreement with theoretical predictions ${ }^{25}$ and experimental observations in $\mathrm{Pt} / \mathrm{Co} / \mathrm{MgO},{ }^{10} \mathrm{Pt} / \mathrm{Co} / \mathrm{Al}_{2} \mathrm{O}_{3},{ }^{15}$ and $\mathrm{Pt} / \mathrm{Co} / \mathrm{HfO}_{2}{ }^{14}$ structures.

\section{ACKNOWLEDGMENTS}

We gratefully acknowledge funding from the French National Research Agency (ANR) under the project ANR2010-BLANC-1006-ELECMADE and from the Nanosciences Foundation of Grenoble. FePt-based device fabrication was carried out at the Nanofab cleanroom facilities in Grenoble.

\section{REFERENCES}

1. S. S. P. Parkin, K. P. Roche, M. G. Samant, P. M. Rice, R. B. Beyers, R. E. Scheuerlein, E. J. O' Sullivan, S. L. Brown, J. Bucchigano, D. W. Abraham, Yu Lu, M. Rooks, P. L. Trouilloud, R. A. Wanner, W. J. Gallagher, 'Exchange-biased magnetic tunnel junctions and application to nonvolatile magnetic random access memory' J. Appl. Phys. 85, 5828 (1999).

2. S. Gider,B.-U. Runge, A. C. Marley, S. S. P. Parkin 'The Magnetic Stability of Spin-Dependent Tunneling Devices' Science 281, 797 (1998).

3. H. Li, Y. Chen 'An overview of non-volatile memory technology and the implication for tools and architectures' Design, Automation Test in Europe Conference Exhibition, 2009. DATE ó9 731-736 (2009).

4. A. Driskill-Smith, D. Apalkov, V Nikitin, X. Tang, S. Watts, D. Lottis, K. Moon, A. Khvalkovskiy, R. Kawakami, X. Luo, A. Ong, E. Chen, M. Krounbi, 'Latest Advances and Roadmap for In-Plane and Perpendicular STT-RAM' Memory Workshop (IMW), 2011 3rd IEEE International 1-3 (2011).

5. H. Ohno, D. Chiba, F. Matsukura, T. Omiya, E. Abe, T. Dietl, Y. Ohno, K. Ohtani, 'Electric-field control of ferromagnetism' Nature 408, 944 (2000).

6. H. Ohno, 'A window on the future of spintronics' Nat. Mater. 9, 952 (2010).

7. M. Weisheit, S. Fähler, A. Marty, Y. Souche, C. Poinsignon, D. Givord,'Electric Field-Induced Modification of Magnetism in Thin-Film Ferromagnets' Science 315, 349-351 (2007).

8. T. Maruyama, Y. Shiota, T. Nozaki, K. Ohta, N. Toda, M. Mizuguchi, A. A. Tulapurkar, T. Shinjo, M. Shiraishi, S. Mizukami, Y. Ando, Y. Suzuki, 'Large voltage-induced magnetic anisotropy change in a few atomic layers of iron' Nat. Nano. 4, 158-161 (2009).

9. M. Endo, S. Kanai, S. Ikeda, F. Matsukura, H. Ohno, 'Electric-field effects on thickness dependent magnetic anisotropy of sputtered $\mathrm{MgO} / \mathrm{Co}_{40} \mathrm{Fe}_{40} \mathrm{~B}_{20} /$ Ta structures' Appl. Phys. Lett. 96, 212503 (2010).

10. D. Chiba, S. Fukami, K. Shimamura, N. Ishiwata, K. Kobayashi, T. Ono, 'Electrical control of the ferromagnetic phase transition in cobalt at room temperature' Nat. Mater. 10, 853856 (2011).

11. K. Shimamura, D. Chiba, S. Ono, S. Fukami, N. Ishiwata, M. Kawaguchi, K. Kobayashi, T. Ono, 'Electrical control of Curie temperature in cobalt using an ionic liquid film' Appl. Phys. Lett. 100, 122402 (2012).

12. W.-G. Wang, M. Li, S. Hageman, C. L. Chien, 'Electric-field-assisted switching in magnetic tunnel junctions' Nat. Mater. 11, 64-68 (2012).

13. A. A. Allwood, G. Xiong, C. C. Faulkner, D. Atkinson, D. Petit, R. P. Cowburn, 'Magnetic Domain-Wall Logic' Science 309, 1688-1692 (2005).

14. A.J. Schellekens, A. van den Brink, J.H. Franken, H.J.M. Swagten, B. Koopmans, 'Electric-field control of domain wall motion in perpendicularly magnetized materials' Nat. Com. 3, 847 (2012).

15. D. Chiba, M. Kawaguchi, S. Fukami, N. Ishiwata, K. Shimamura, K. Kobayashi, T. Ono, 'Electric-field control of magnetic domain-wall velocity in ultrathin cobalt with perpendicular magnetization' Nat. Com. 3, 888 (2012). 
16. W. Lin, N. Vernier, G. Agnus, N. Lei, S. Eimer, D. Ravelosona., 'Interfacial charge accumulation effect on magnetic domain wall nucleation and propagation in a $\mathrm{Pt} / \mathrm{Co} / \mathrm{Pt} / \mathrm{Al} 2 \mathrm{O} 3$ structure' (submitted), arXiv:1201.5917v2 [cond-mat.mtrl-sci] (2012).

17. A. Bernand-Mantel, L. Herrera-Diez, L. Ranno, S. Pizzini, J. Vogel, D. Givord, O. Boulle, I. M. Miron, S. Auffret, G. Gaudin, 'Electric-field control over domain wall nucleation near the spin reorientation transition in a metallic ferromagnet' (submitted), (2012).

18. A. W. Rushforth, E. De Ranieri, J. Zemen, J. Wunderlich, K. W. Edmonds, C. S. King, E. Ahmad, R. P. Campion, C. T. Foxon, B. L. Gallagher, K. Výborný, J. Kučera, T. Jungwirth, 'Voltage control of magnetocrystalline anisotropy in ferromagnetic-semiconductor-piezoelectric hybrid structures' Phys. Rev. B 78, 085314 (2008).

19. N. Lei, T. Devolder, G. Agnus, P. Aubert, L. Daniel, J.-V. Kim, W. Zhao, C. Chappert, D. Ravelosona, P. Lecoeur, 'Electric field control of domain wall logic in piezoelectric/ferromagnetic nanodevices' (submitted), arXiv:1201.4939v1 [cond-mat.mtrl-sci] (2012).

20. G. D. Wilk, R. M. Wallace, J. M. Anthony, 'High-k gate dielectrics: Current status and materials properties considerations' J. Appl. Phys. 89, 5243 (2001).

21. M. J. Biercuk, D. J. Monsma, C. M. Marcus, J. S. Becker, R. G. Gordon, 'Low-temperature atomic-layerdeposition lift-off method for microelectronic and nanoelectronic applications' Appl. Phys. Lett. 83, 2405 (2003).

22. P. Gonon, M. Mougenot, C. Vallée, C. Jorel, V. Jousseaume, H. Grampeix, F. El Kamel, 'Resistance switching in HfO2 metal-insulator-metal devices' J. Appl. Phys. 107, 074507 (2010).

23. T. Seki, M. Kohda, j. Nitta, K. Takanashi, 'Coercivity change in an FePt thin layer in a Hall device by voltage application' Appl. Phys. Lett. 98, 212505 (2011).

24. L. Herrera Diez, A. Bernand-Mantel, O. Michele, L. Ranno, D. Givord, L. Vila, P. Warin, A. Marty, 'Electric-field effect on coercivity distributions in FePt magneto-electric devices' (submitted), (2012).

25. H. Zhang, M. Richter, K. Koepernik, I. Opahle, F. Tasnádi, and H. Eschrig, 'Electric-field control of surface magnetic anisotropy: a density functional approach' New J. Phys. 11, 043007 (2009). 